\title{
Search Engines for Shape Selectivity
}

\author{
James Wei · Christodoulos A. Floudas • \\ Chrysanthos E. Gounaris · Gabor A. Somorjai
}

Received: 27 August 2009/Accepted: 27 August 2009/Published online: 23 September 2009

(C) The Author(s) 2009. This article is published with open access at Springerlink.com

\begin{abstract}
Zeolites have been used successfully for over 30 years to separate small molecules of almost identical physical and chemical properties, relying on shape differences such as normal from branched paraffins. Many zeolites have approximately circular windows and these are the only ones used in current applications. As of today, the structures of 176 zeolites are available; many of them have windows that may be described as elliptic or irregular, presenting many opportunities for shape selective catalysis or adsorption. A molecule approaching a window may take a number of orientations, and it is difficult to visualize the three-dimensional shape and the best fit to the window. Search engines are needed to accelerate the discovery of one or more zeolite windows that can separate a given pair of molecules. A great deal of progress has already been made to construct such search engines, and innovative industrial processes may follow in the future.
\end{abstract}

Keywords Shape selectivity - Zeolites .

Molecular flexibility $\cdot$ Strain energy

The shapes of molecules have been utilized for catalysis [1] and for industrial separations [2] by zeolites. Shape selective separation is particularly useful when two molecules are closely related in physical and chemical properties but have different shapes, such as the case of normal and branched paraffins in the catalytic de-waxing by

J. Wei $(\bowtie)$. C. A. Floudas · C. E. Gounaris · G. A. Somorjai Department of Chemical Engineering, Princeton University, Princeton, NJ 08544, USA

e-mail: jameswei@princeton.edu

G. A. Somorjai

e-mail: somorjai@berkeley.edu
SAPO-11. A linear normal paraffin can be rotated to a slim configuration so that its projection onto the plane of the window, or "footprint," is small enough to penetrate the window with ease. On the other hand, a branched paraffin has a footprint that is larger regardless of the rotation angles $[3,4]$. Another industrial example is the separation of the narrower $p$-xylene from the wider $o$ - and $m$-xylenes by ZSM-5. Such a separation can be due to one of three mechanisms: steric separation is achieved when only small and properly shaped molecules can diffuse in the pores but larger molecules are excluded; equilibrium separation is achieved when the equilibrium adsorption concentrations are selectively reduced by different amounts; and kinetic separation is achieved when one molecule adsorbs faster than the other due to different speeds of diffusion.

\section{Hard Sphere Models and Footprints}

The $x-y-z$ coordinates of all the atoms in 176 zeolites are given in the Atlas of Zeolite Framework Types [5]. Figure 1 shows that some of the zeolite windows are approximately circular, such as the 8-ring LTA, the 10-ring MFI, and the 12-ring FAU. The CGF is a highly eccentric ellipse, and the RON is nearly square, while the CZP is irregular. WEN and CLO have intrusions jutting into the center cavity, thus creating C-shaped and clover shaped openings. The simplest model for the entry of a molecule is to assume that all the atoms on the window and the molecule are hard spheres, so that the possibility of penetration is simply a matter of geometry.

It is easy to search for a circular window that would separate two spherical molecules of different diameters. For instance the noble gases vary in their van der Waals diameters from $2.5 \AA$ for helium to $4.0 \AA$ for xenon, so a 
Fig. 1 Variety of shapes exhibited by zeolite windows. Size of depicted windows ranges between 8 and 24 O-atoms

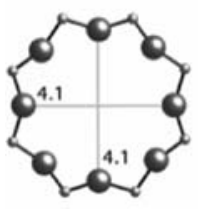

LTA

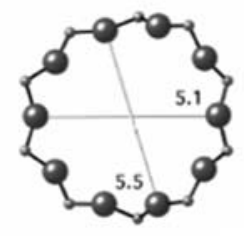

MFI

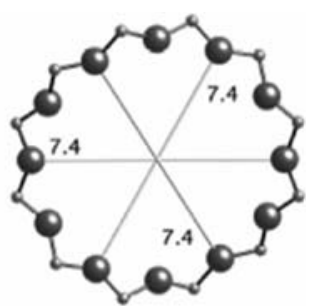

FAU
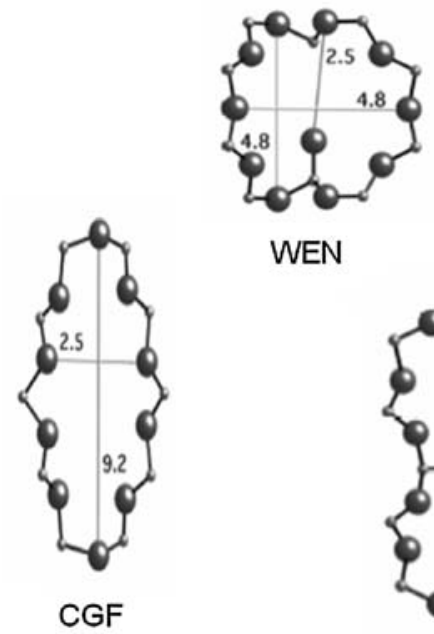

WEN
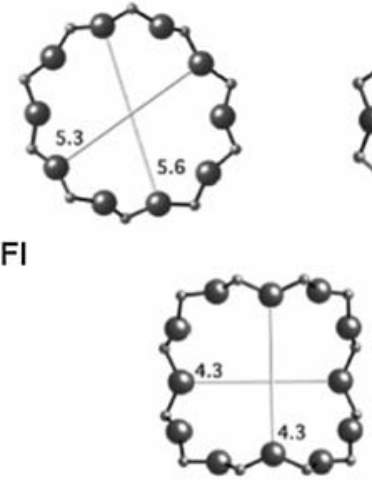

RON
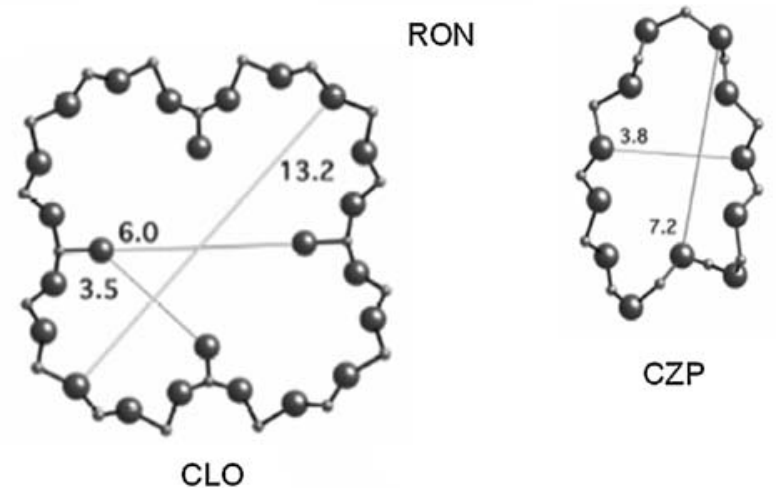

3A zeolite with a window diameter of $3.0 \AA$ will allow the passage of helium and neon, but retain argon, krypton and xenon. However, most molecules are not spheres. Molecules with $\mathrm{C}_{\infty \mathrm{v}}$ symmetry, such as $\mathrm{HCl}$ and $\mathrm{CO}$, and with $\mathrm{D}_{\infty \mathrm{h}}$ symmetry, such as hydrogen and acetylene, do produce circular footprints [6]. The optimal rotation is to point the narrowest footprint towards the window; that is, to place the molecular axis perpendicular to the window plane.

The coordinates of each atom in a molecule can be computed with a quantum chemistry software such as the HyperChem [7], while the van der Waals radii can be used as effective radii for each atom. A mathematical optimization-based computer program has been developed to rotate a molecule in three directions $(\varphi, \theta, \psi)$ and see if it will clear a given window [4]. For an elongated linear molecule, such as $n$-decane, the projection on the window plane can take many forms, three of which are shown in Fig. 2. Clearly, form (a) has the smallest footprint and would be the most promising configuration to assume in order to penetrate a window. In fact, all the normal paraffins in the all-trans configuration have the same footprint, which consists of only two overlapping carbon, one central hydrogen, and four peripheral hydrogen atoms.

In an improved rigid ellipse model, the rigid circular windows would be replaced by elliptical ones, so that a single diameter is replaced by a pair of major and minor diameters. Figure 3 depicts the footprints of the three

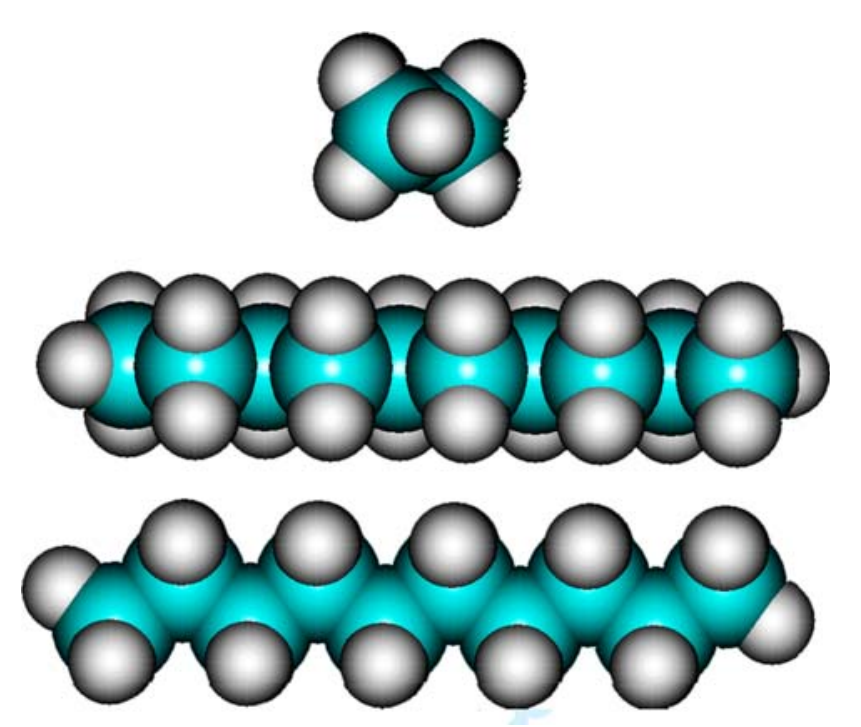

Fig. 2 Three projections of $n$-decane in the all-trans configuration. Projection (a) corresponds to the smallest footprint and is the most suitable for penetration

pentanes; the first footprint (a) corresponds to $n$-pentane in the perpendicular orientation, showing only two carbon nuclei-consistent with Fig. 2. Neo-pentane (c) has a much larger footprint compared to isopentane (b) which, in turn, has a larger footprint than $n$-pentane (a).

Figure 4 shows the major and minor diameters of the footprints of a number of molecules. Notice that the normal 
Fig. 3 The footprints of the $\mathrm{C}_{5}$ paraffins: a $n$-pentane, b $i$-pentane, c neo-pentane. The dots correspond to the atomic nuclei and the circles to the van der Waals electron radii
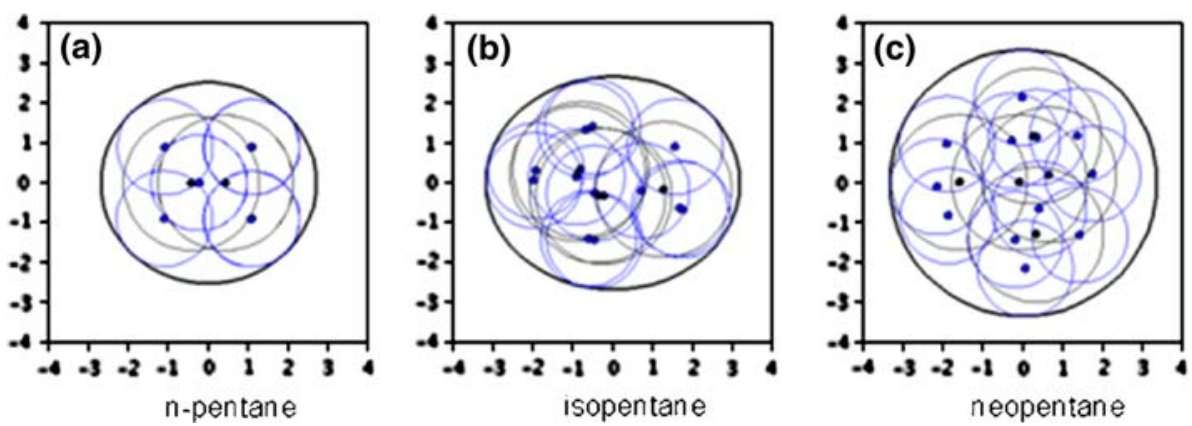

(d) The bond angles and bond lengths of the molecules are also flexible; this is a much more complex problem as there are many more types of bonds to consider.

(e) The entropy effects due to the possibility that the approach of a molecule towards the window is turned back, resulting to the same molecule coming back for another approach.

The parameter values used are given in Table 1, and are derived from empirical data of gas compressibility and from transport properties [8-10].

$$
\begin{aligned}
& E_{d}=4 \varepsilon\left[\left(\frac{\sigma}{\rho}\right)^{12}-\left(\frac{\sigma}{\rho}\right)^{6}\right] \\
& E_{Z}=\sum \frac{k_{\mathrm{TO}}}{2}\left(d_{\mathrm{TO}}-d_{\mathrm{TO}, 0}\right)^{2}+\sum \frac{k_{\mathrm{OO}}}{2}\left(d_{\mathrm{OO}}-d_{\mathrm{OO}, 0}\right)^{2}
\end{aligned}
$$

We discovered rather early that, when a complex molecule approaches windows of different shapes, they may assume different footprints to achieve the best penetration. Therefore, instead of presenting a unique footprint for each molecule, we have a different footprint for each molecule-window combination. Starting with model (b), each time we squeeze a molecule into a

\begin{tabular}{|c|c|c|c|c|c|c|c|c|}
\hline \multicolumn{9}{|c|}{ Atomic van der Waals radii in $\AA$ [8] } \\
\hline Atom & $\mathrm{H}$ & $\mathrm{C}$ & $\mathrm{N}$ & $\mathrm{O}$ & $\mathrm{F}$ & & S & $\mathrm{U}$ \\
\hline$r_{\mathrm{vdw}}$ & 1.20 & 1.70 & 1.50 & 1.40 & 1.35 & & 1.85 & 1.86 \\
\hline \multicolumn{9}{|c|}{ Molecular Lennard-Jones parameters, $\varepsilon / R$ and $\sigma$ [9] } \\
\hline \multicolumn{2}{|c|}{ Species He } & $\mathrm{Ne} \quad \mathrm{Ar}$ & $\mathrm{H}_{2} \quad \mathrm{~N}_{2}$ & $\mathrm{O}_{2}$ & $\mathrm{CO} \mathrm{CO}_{2}$ & ${ }_{2} \mathrm{CH}_{4}$ & $\mathrm{I}_{4} \mathrm{C}_{2} \mathrm{H}_{4}$ & $\mathrm{C}_{2} \mathrm{H}_{6}$ \\
\hline$\varepsilon / R(\mathrm{~K})$ & 10.22 & 35.6120 & $\begin{array}{ll}37 & 95.1\end{array}$ & 118 & 100189 & 149 & 9199 & 243 \\
\hline$\sigma(\mathrm{pm})$ & 256 & 341 & 293370 & 358 & 376449 & 378 & 8452 & 395 \\
\hline \multicolumn{9}{|c|}{$\mathrm{T}-\mathrm{O}-\mathrm{T}$ bond length and bond angle parameters [10] } \\
\hline \multicolumn{4}{|c|}{$k_{\mathrm{TO}}\left(\mathrm{kJ} / \mathrm{mol} \AA^{2}\right)$} & \multicolumn{5}{|c|}{2,092} \\
\hline \multicolumn{4}{|c|}{$k_{\mathrm{OO}}\left(\mathrm{kJ} / \mathrm{mol} \AA^{2}\right)$} & \multicolumn{5}{|c|}{430} \\
\hline
\end{tabular}

Table 1 Parameter values used 
window, the radii of some atoms are shortened which requires an activation energy. We define $\mathrm{E}$ to be the total activation energy by summing all the energies involved in squeezing all the molecular and window atoms, however only the $E_{d}$ is used in the first model.

$E=\sum E_{d}+\sum E_{\ell}+\sum E_{\theta}$

There is a cost associated with this energy, which will have the effect of lowering the diffusion coefficient, as well as depressing the equilibrium adsorption concentration according to the Boltzmann equation.

$\frac{C}{C_{0}}=\exp \left(-\frac{E}{\mathrm{RT}}\right)$

Figure 5 shows the values of $C / C_{0}$ at different temperature and values of $E$. For a non-zero value of $E$, the concentration at absolute zero temperature is always zero, but the concentration increases with temperature. When the energy penalty is small, such as the case of (E/RT) $<0.1$ (e.g., $E=0.5 \mathrm{~kJ} / \mathrm{mol}$ and $T=500 \mathrm{~K}$ ), the equilibrium concentration ratio is only slightly depressed to a value no smaller than 0.905 . But when $(E / \mathrm{RT})>3$ (e.g., $E=16 \mathrm{~kJ} / \mathrm{mol}$ and $T=500 \mathrm{~K}$ ), the $C / C_{0}$ ratio is lowered to less than 0.050 . When $E>50 \mathrm{~kJ} / \mathrm{mol}$, there is not much adsorption even at 1,000 $\mathrm{K}$.

Figure 6 shows a table of six aromatic molecules from benzene to ethyl-benzene, approaching seven windows of 12-ring zeolites. These are LTL for Linde Type L, MAZ for Mazzite, MEI for ZSM-18, MOR for Mordenite, MTW for ZSM-12, OSI for UiO-6, and RON for Roggianite.

When a column is all bright green $(E / \mathrm{RT}<0.001)$ such as for LTL or MAZ, all six aromatic molecules can penetrate easily, so there is no separation. When the entire column is red $(E / \mathrm{RT}>7)$, such as the case of RON, none of the six aromatic molecules can penetrate, so again there

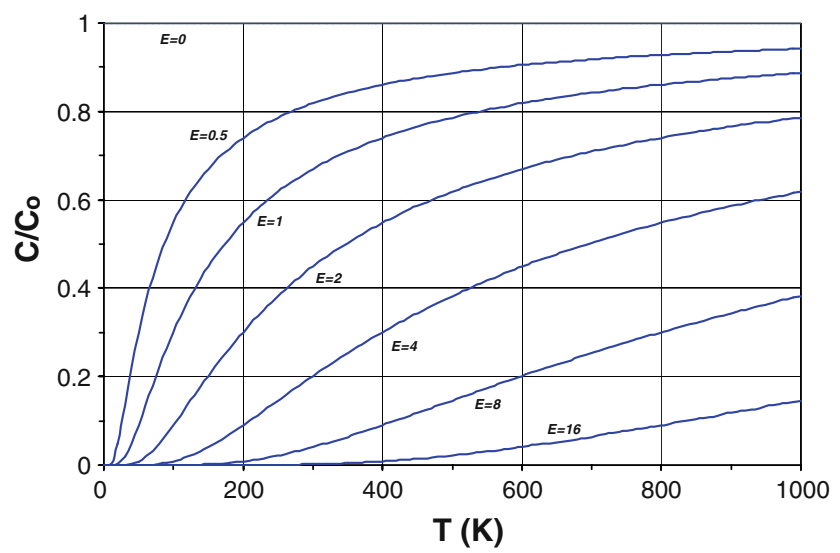

Fig. 5 The Boltzmann reduction in equilibrium concentration $C / C_{0}$ at different temperatures and for different values of activation energy $E$ (in $\mathrm{KJ} / \mathrm{mol}$ )

\begin{tabular}{|c|c|c|c|c|c|c|c|c|}
\hline 19 & 1.000 & 1.000 & 1.000 & 1.000 & 1.000 & 1.000 & 0.000 & BENZ \\
\hline 20 & 1.000 & 1.000 & 1.000 & 1.000 & 1.000 & 1.000 & 0.000 & TOL \\
\hline 21 & 1.000 & 1.000 & 0.076 & 0.321 & 0.000 & 0.000 & 0.000 & $O-X Y L$ \\
\hline 22 & 1.000 & 1.000 & 0.224 & 0.705 & 0.000 & 0.000 & 0.000 & $M-X Y L$ \\
\hline 23 & 1.000 & 1.000 & 1.000 & 1.000 & 1.000 & 1.000 & 0.000 & $P-X Y L$ \\
\hline \multirow[t]{3}{*}{24} & 1.000 & 1.000 & 1.000 & 1.000 & 0.200 & 0.831 & 0.000 & $E-B E N$ \\
\hline & LTL & MAZ & MEI & MOR & MTW & OSI & RON & \\
\hline & & 0 & $0.0-0.3$ & $0.3-0.7$ & $0.7-1.0$ & 1 & & \\
\hline
\end{tabular}

Fig. $6 C / C_{0}$ achieved by six aromatic molecules as they penetrate select zeolite windows $(T=300 \mathrm{~K})$

is no separation. But, for MTW with a 12-ring opening that measures $5.6 \times 6.0 \AA{ }^{1}$ there is easy passage for benzene, toluene and $p$-xylene, limited passage for ethyl-benzene, and no passage for $o$ - and $m$-xylene. Figure 7 shows the complete $C / C_{0}$ map for 38 molecules and 217 windows. There are 7 small molecules from hydrogen to ammonia, 11 paraffins and olefins, 6 aromatics, 11 biological molecules, and 3 special symmetrical molecules. The smaller molecules are placed at the top, while the larger ones are placed at the bottom. The 217 zeolite windows are presented in order of increasing size of aperture. The smaller ones (e.g., 7 O-atom rings) are placed on the left of the map, while the larger rings (e.g., CLO with $24 \mathrm{O}$-atoms) are placed towards the right. The upper right corner is all green, corresponding to the easy passage of small molecules through large windows; the lower left corner is all red corresponding to small windows denying entry to large molecules. From the point of view of selective separation, the most interesting parts of the map are the green and yellow border regions in between.

The power of a zeolite to separate molecules A and B can be measured by the selectivity parameter $\gamma_{\mathrm{AB}}$, which is defined to be.

$\gamma_{\mathrm{AB}}=\left|\left(\frac{C}{C_{0}}\right)_{\mathrm{A}}-\left(\frac{C}{C_{0}}\right)_{\mathrm{B}}\right|=\left|\exp \left(-\frac{E_{\mathrm{A}}}{\mathrm{RT}}\right)-\exp \left(-\frac{E_{\mathrm{B}}}{\mathrm{RT}}\right)\right|$

When the two energies are nearly the same, there is no separation and $\gamma_{\mathrm{AB}}=0$; when the two energies are widely different, there is good separation and selectivity approaches the value of $\gamma_{\mathrm{AB}}=1$. The value of $\gamma_{\mathrm{AB}}$ depends on the temperature $T$ and can go through a maximum at

$T^{*}=\frac{1 E_{\mathrm{A}}-E_{\mathrm{B}}}{k \ln \left(E_{\mathrm{A}} / E_{\mathrm{B}}\right)}=\frac{E_{\mathrm{LM}}}{k}$

where $E_{\mathrm{LM}}$ is the logarithmic mean of the two energies.

\footnotetext{
${ }^{1}$ Openings are reported in terms of distances between nuclei of $\mathrm{O}$-atoms. Actual openings are, thus, smaller by two $\mathrm{O}$-atom radius (typically $1.40 \AA$ ).
} 
Fig. 7 Map of $C / C_{0}$ values for 38 molecules and 217 windows $(T=300 \mathrm{~K})$. The numbers at the bottom of the horizontal axis refer to the number of $\mathrm{O}$-atoms on the zeolite windows

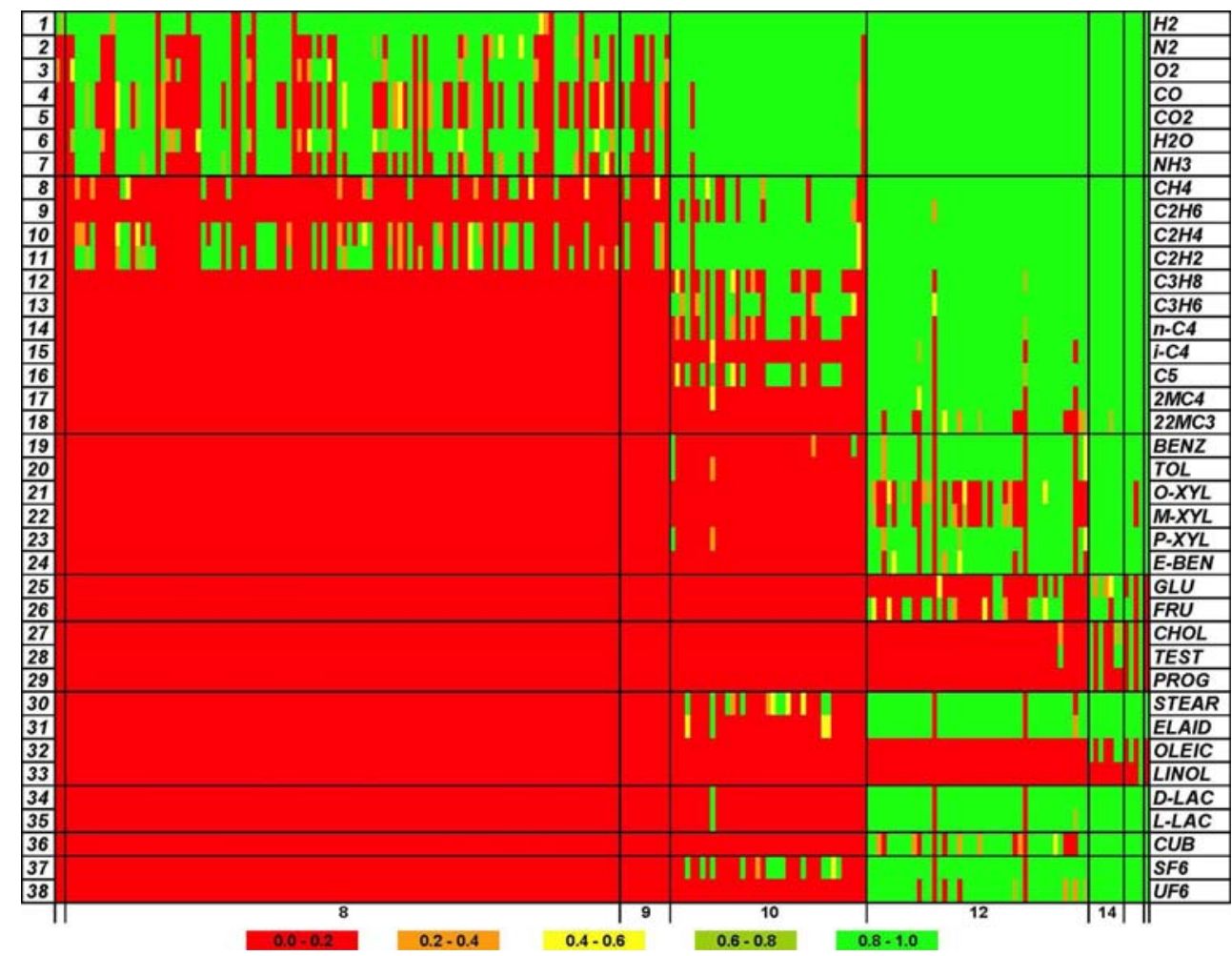

Consider two molecules A and B, and assume that we have the task to find the best zeolite window to separate the two. We can search the computed table of activation energies, and look for windows that have low $E_{\mathrm{A}}$ (zero is preferred), as well as high $E_{\mathrm{B}}$. From such a screening of the complete set of windows, we form a small set of potential winners for further investigation on, e.g., what is the best temperature for separation, availability, and cost of these zeolites. Figure 8 exhibits the four typical selectivitytemperature trends in the separation of ethane (elliptical footprint $3.95 \times 3.40 \AA)$ from ethylene $(3.40 \times 3.40 \AA)$. For the RUB-3 (RTE) zeolite with windows of size $4.4 \times 3.7 \AA$, the activation energies are $E_{\mathrm{A}}=0$ for ethylene and $E_{\mathrm{B}}=26.7 \mathrm{~kJ} / \mathrm{mol}$ for ethane. The separation factor $\gamma_{\mathrm{AB}}$ is close to 1 at any temperature, so RTE is a very desirable candidate. For the more eccentric AlPO-H2 (AHT) with its $6.8 \times 3.3 \AA$ windows, we have $E_{\mathrm{A}}=0$ and $E_{\mathrm{B}}=5.0 \mathrm{~kJ} / \mathrm{mol}$. Separation is perfect at very low temperatures as A can enter freely but B cannot enter at all; but when the temperature rises, $\mathrm{B}$ begins to gain enough thermal energy to enter the pores, resulting in reduced separation. The AlPO-22 zeolite (AWW) has symmetric openings of size $3.9 \times 3.9 \AA$, and corresponds to energy values of $E_{\mathrm{A}}=4.2$ and $E_{\mathrm{B}}=49.8(\mathrm{~kJ} / \mathrm{mol})$. In this case, even A needs sufficiently high temperature for entry; the separation improves with temperature till it reaches a maximum (occurring here beyond the depicted range of $T$ ), then $\mathrm{B}$ begins to penetrate faster and the separation worsens. Finally, in the case of RUB-24 (RWR), with narrow windows of size $5.0 \times 2.8 \AA$ and activation energies $E_{\mathrm{A}}=19.7$ and $E_{\mathrm{B}}=268(\mathrm{~kJ} / \mathrm{mol})$, neither A nor B can penetrate in the temperature range below $900 \mathrm{~K}$.

The best separation is reached when $\mathrm{A}$ has free entry and $\mathrm{B}$ is hindered; that is, $E_{\mathrm{A}}=0$ and $E_{\mathrm{B}}>8 \mathrm{~kJ} / \mathrm{mol}$, so that a very sharp separation $\left(\gamma_{\mathrm{AB}}>0.95\right)$ can be carried out at room temperature. However, if the entry of both $\mathrm{A}$ and $\mathrm{B}$ are hindered, to reach a separation selectivity of $\gamma_{\mathrm{AB}}=0.2$ we would recommend zeolites that correspond to activation

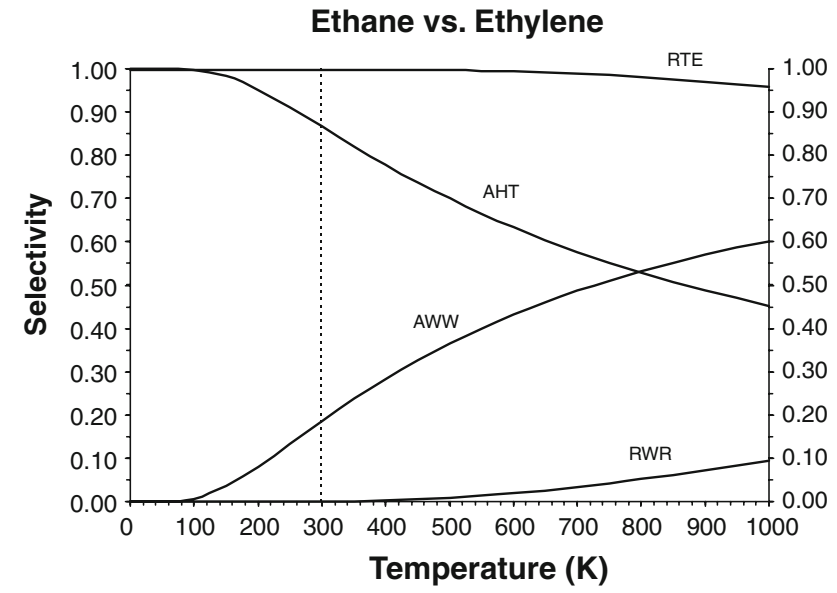

Fig. 8 Separation selectivity, as a function of temperature, for four zeolites. Here, A is ethylene and B is ethane 
energy values $E_{\mathrm{A}}<1$ and $E_{\mathrm{B}}>2(\mathrm{~kJ} / \mathrm{mol})$. If a higher separation selectivity of $\gamma_{\mathrm{AB}}=0.8$ is desired, we would recommend $E_{\mathrm{A}}<0.1$ and $E_{\mathrm{B}}>5(\mathrm{~kJ} / \mathrm{mol})$.

\section{Model Refinements}

During our computations, it is important to use appropriately tuned values for the atom radii parameters. It should be noted that the van der Waals values were derived from viscosity data, and the Lennard-Jones values were derived from equations of state and gas compressibility data; thus, they are not necessarily the best to describe the interaction of molecules in contact with walls. Particularly questionable is the value of $1.40 \AA$ for oxygen atomic radius, as it would predict that aromatic molecules could not penetrate ZSM-5, which is not the case. We have studied the effect of atomic radius of oxygen on the penetration of the three xylenes in ZSM-5, and concluded that the appropriate radius is actually between 0.95 and $1.10 \AA$ (so that the windows become larger and more accommodating). On the other hand, the penetration of nitrogen and methane in Sr-ETS-4 at $573 \mathrm{~K}$ would be better explained if the effective radius is $1.30-1.40 \AA$ [11].

The next model improvement is to add the $\mathrm{T}-\mathrm{O}-\mathrm{T}$ bond angle and $\mathrm{T}-\mathrm{O}$ bond length flexibilities of the windows. When these flexibilities are included, the activation energy is reduced from $E_{\text {rig }}$ (rigid) to $E_{\text {flex }}$ (flexible), and the effect can be compared by the ratio $E_{\text {flex }} / E_{\text {rig }}$, which is the slope of the data presented in Fig. 9. This ratio can vary from 0.3 to 1.0 , and seems to center around the value of 0.75 . The

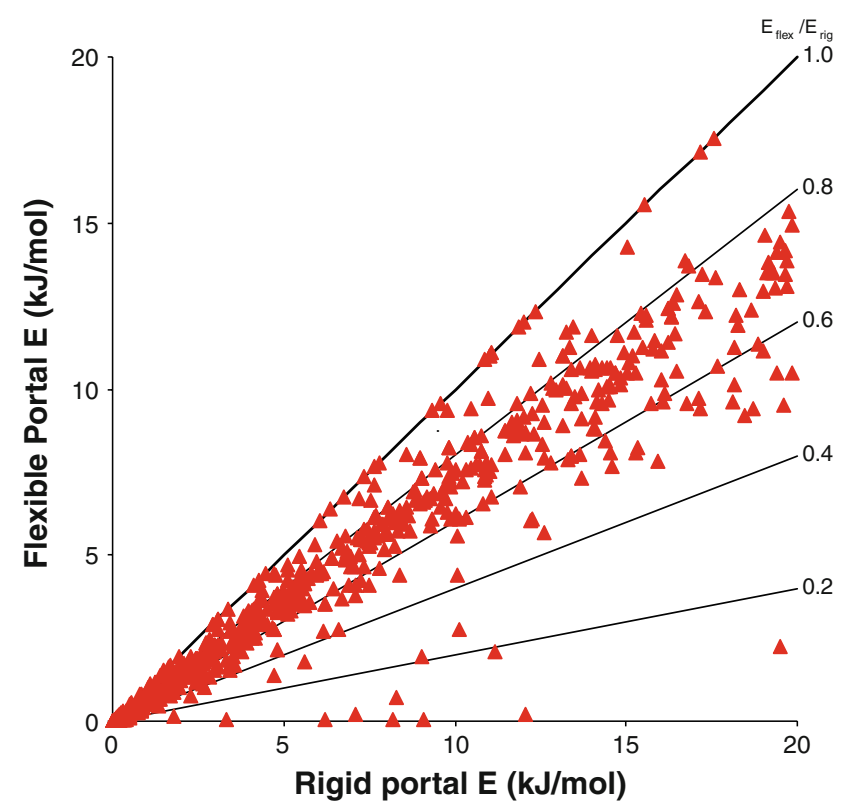

Fig. 9 Comparing activation energies for flexible window bond angles and lengths with the corresponding activation energies for rigid window bonds parameter values of the spring, taken from [10], are derived from diffusion data for $\mathrm{NaA}$ zeolite; their validity for other zeolites is not guaranteed.

Commercial uses of zeolites always involve several modification steps to enhance selectivity, such as by changing the cation from sodium to calcium, by polymer additives, by coke deposition, and by steaming. All of these would have effects on the size and shape of window opening, and consequently the activation energy and equilibrium concentrations.

\section{Future Opportunities}

We can retrace our steps on the classical zeolites used in current processes, and see if there are better candidates than the ones currently used on industrial scale, such as in the separation of normal to branched paraffins, and in separating $o$ - and $m$-xylenes from $p$-xylene. However, future opportunities more probably lie in separations that have not yet been developed or optimized so extensively. Recently, we had success in finding a suitable zeolite to remove a set of fermentation inhibitors from ligno-cellulosic biomass hydrolysis [12]. The compounds furfural, 5-hydroxymethylfurfural (HMF), and vanillin are produced in fermentation and are inhibitors that depress enzyme activity and the production of ethanol. We searched for a highly hydrophobic adsorbent that excludes vanillin while adsorbing HMF and furfural, and a second adsorbent that excludes both vanillin and HMF, but adsorbs furfural. The search predicted a table of values for $C / C_{0}$, and a few are shown in Table 2. Subsequently, experiments were performed on the MFI and FER structures, which confirmed that neither would admit vanillin due to its bulk shape; MFI would make some preference for furfural over HMF, but FER would show a very large preference [12].

Table $2 C / C_{0}$ predictions for separators of lingo-cellulosic biomass fermentation inhibitors [11]

\begin{tabular}{lllll}
\hline Molecule & OFF & MFI & FER & STT \\
\hline Furfural & 0.911 & 0.717 & 0.724 & 0.700 \\
HMF & 1.000 & 0.085 & 0.010 & 0.000 \\
Vanillin & 0.000 & 0.000 & 0.000 & 0.000 \\
\hline
\end{tabular}

Table 3 Comparison between zeolites and other classes of sorbent materials

\begin{tabular}{lll}
\hline Material & Diameter, $\AA$ & MW of potential sorbates \\
\hline Zeolite 12-ring & 7.4 & 200 \\
Zeolite 20-ring & 12.7 & 1,000 \\
Nanotube & $3.7-24.0$ & 10,000 \\
MCM-41 & $25-100$ & 400,000 \\
\hline
\end{tabular}




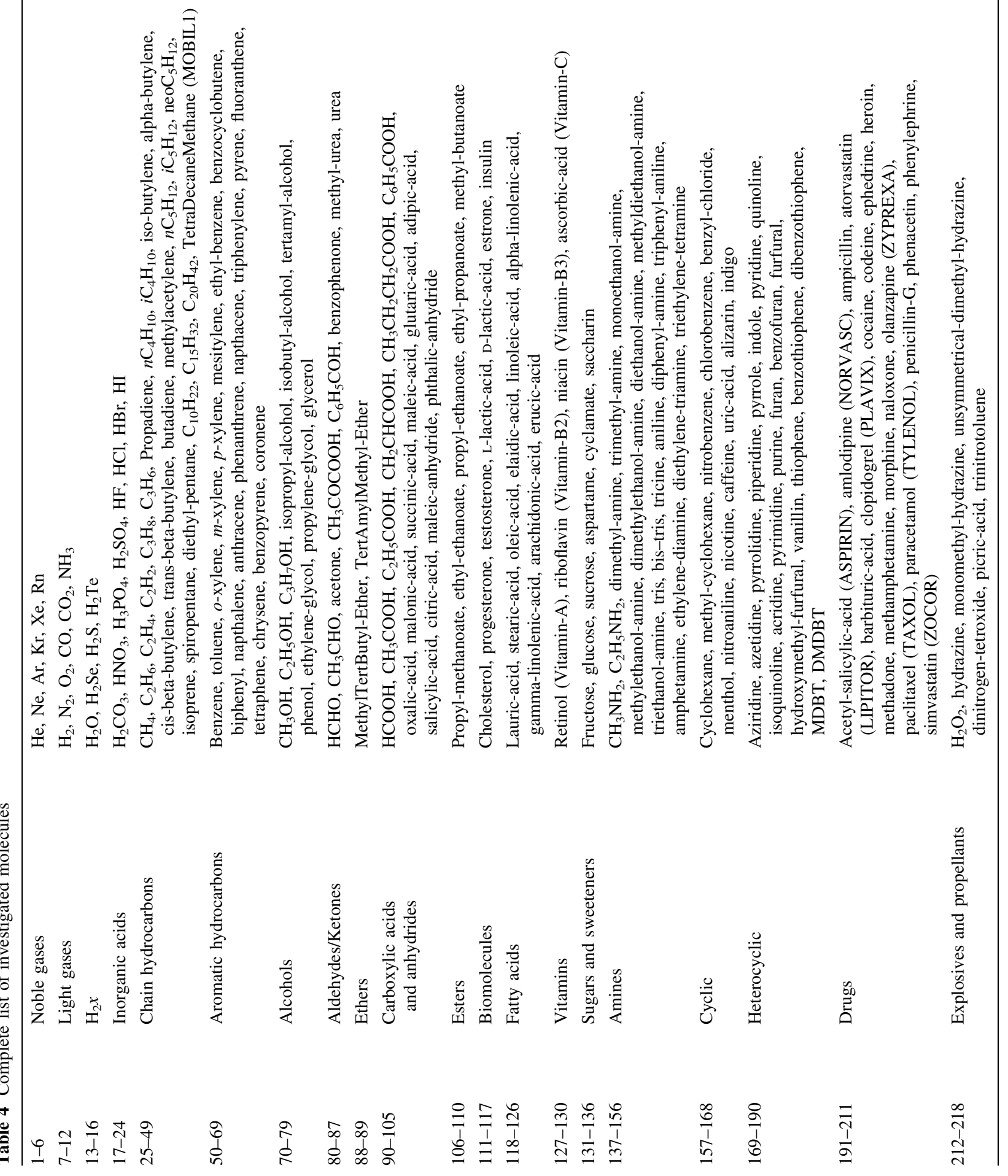


Current industrial applications have been confined to small windows and small molecules. Nowadays, there exists a wealth of newer and larger mesoporous materials that are available for shape selective separations (see Table 3), and the list is growing everyday. They represent tremendous opportunities that are waiting to be exploited.

In preparation for new discoveries, we have enlarged the list of the molecules we investigated, from the original 38 to the 290 molecules listed in Table 4. The expanded list includes many biological and pharmaceutical molecules with molecular weights that are as large as 2,319 (e.g., insulin). We now have the tools to make serious investigations to the following question: "given two sets of molecules that may be difficult to separate by conventional means, are there one or more microporous material that would be appropriate for separation and catalysis?"

Acknowledgments The authors gratefully acknowledge financial support from the National Science Foundation.

Open Access This article is distributed under the terms of the Creative Commons Attribution Noncommercial License which permits any noncommercial use, distribution, and reproduction in any medium, provided the original author(s) and source are credited.

\section{References}

1. Chen NY, Garwood WE, Dwyer FG (1996) Shape selective catalysis in industrial applications. Marcel Dekker, New York

2. Yang R (2003) Adsorbents: fundamentals and applications. Wiley, New York

3. Gounaris CE, Floudas CA, Wei J (2006) Rational design of shape selective separation, catalysis: I. Concepts and analysis. Chem Eng Sci 61:7933-7948

4. Gounaris CE, Wei J, Floudas CA (2006) Rational design of shape selective separation and catalysis: II. Mathematical model and computational studies. Chem Eng Sci 61:7949-7962

5. Baerlocher C, McCusker LB, Olson DH (2007) Atlas of Zeolite framework types, 6th edn, Structure Commission of the International Zeolite Association, Elsevier, Amsterdam, Holland

6. Eliel EL, Wilen SH (1994) Stereochemistry of organic compounds. Wiley, New York

7. Hypercube Inc (1996) HyperChem ${ }^{\circledR}$ Getting Started Release 5.0 for Windows. Gainsville, Florida

8. Pauling L (1947) General chemistry. Dover, New York

9. McQuarrie DA, Simon JD (1999) Molecular thermodynamics. University Science Books, Sausalito

10. Santikary P, Yashonath S (1994) Dynamics of zeolite cage and its effect on the diffusion properties of sorbate. J Phys Chem 98: 9252-9259

11. Gounaris CE, Ranjan R, Tsapatsis M, Wei J, Floudas CA (2009) Rational design of shape selective separation and catalysis: lattice relaxation and effective aperture size. AIChE J (submitted for publication)

12. Ranjan R, Thust S, Gounaris CE, Woo M, Floudas CA, Von Keitz M, Valentas KJ, Wei J, Tsapatsis M (2009) Adsorption of fermentation inhibitors from lignocellulosic biomass hydrolyzates for improved ethanol yield and value-added product recovery. Microporous Mesoporous Mater (in press) 